\title{
Liver Protective Effects of Morinda citrifolia (Noni)
}

\author{
Mian-Ying Wang • Diane Nowicki • Gary Anderson • \\ Jarakae Jensen • Brett West
}

Published online: 4 March 2008

(C) The Author(s) 2008

\begin{abstract}
This study evaluated the protective effects of Noni fruit juice on acute liver injury induced by carbon tetrachloride $\left(\mathrm{CCl}_{4}\right)$ in female Sprague-Dawley (SD) rats. Liver damage (micro-centrilobular necrosis) was observed in animals pretreated with $20 \%$ placebo (drinking water) + $\mathrm{CCl}_{4}$. However, pretreatment with $20 \%$ Noni juice in drinking water $+\mathrm{CCl}_{4}$ resulted in markedly decreased hepatotoxic lesions. Furthermore, serum alanine aminotransferase and aspartate aminotransferase levels were significantly lower in the Noni group than the placebo group. In a correlative time-dependent study, one dose of $\mathrm{CCl}_{4}(0.25 \mathrm{~mL} / \mathrm{kg}$ in corn oil, p.o. $)$ in female $\mathrm{SD}$ rats, pretreated with $10 \%$ placebo for 12 days, caused sequential progressive hepatotoxic lesions over a $24 \mathrm{~h}$ period, while a protective effect from $10 \%$ Noni juice pretreatment was observed. These results suggest that Noni juice is effective in protecting the liver from extrinsic toxin exposure.
\end{abstract}

Keywords Morinda citrifolia $\cdot$ Noni $\cdot$ Liver protection . Carbon tetrachloride

\section{Abbreviations \\ ALT alanine aminotransferase \\ AST aspartate aminotransferase}

M.-Y. Wang $(\varangle) \cdot$ D. Nowicki $\cdot$ G. Anderson

Department of Pathology,

University of Illinois College of Medicine,

1601 Parkview avenue,

Rockford, IL 61107, USA

e-mail: mianwang@uic.edu

J. Jensen · B. West

Research and Development, Morinda Holdings Inc.,

Provo, UT 84604, USA
$\mathrm{CCl}_{4} \quad$ carbon tetrachloride

$\mathrm{H} \& \mathrm{E}$ hematoxylin and eosin

LM light microscope

LPO lipid peroxide

SAR superoxide anion radical

TNJ Tahitian Noni ${ }^{\circledR}$ Juice

\section{Introduction}

Carbon tetrachloride $\left(\mathrm{CCl}_{4}\right)$ is a common environmental pollutant and liver carcinogen. Due to its detoxifying function in protecting the body, the liver is a target for $\mathrm{CCl}_{4}$ toxicity [1]. Antioxidants and anti-inflammatory agents play a critical role in liver protection by scavenging superoxide anion radicals (SAR) and neutralizing lipid peroxides (LPO). They also suppress the inflammatory response [2-4]. Therefore, there is need for a natural product that protects the liver, but is also cost-effective and safe, without long-term side effects. This study evaluated the liver protective potential of the fruit juice of Morinda citrifolia (Noni), from Tahiti, a strong antioxidant and anti-inflammatory nutritional supplement.

We have previously reported that Noni juice is an effective antioxidant, demonstrating a dose-dependent effect against SAR and LPO in vitro [5]. Based on the data from our laboratory and others, we suggested that Noni juice may be effective in protecting the liver against acute $\mathrm{CCl}_{4}$ toxicity. To test our hypothesis, a classic $\mathrm{CCl}_{4}$-induced liver injury model was chosen to study the liver protective effect in female SD rats. Morphological change is the most direct sign of liver injury. Histopathological changes were evaluated for morphological evidence of the liver protective properties of Noni juice. Serum alanine aminotransferase 
(ALT) and aspartate aminotransferase (AST) assays that measure liver function were performed on the Noni group $\left(\mathrm{Noni}+\mathrm{CCl}_{4}\right)$ and compared to those of the positive control group (placebo $+\mathrm{CCl}_{4}$ ).

\section{Materials and Methods}

Materials

Carbon tetrachloride was purchased from Sigma Chemical Co., St. Louis, MO, USA. Noni juice was made by Morinda Holdings Inc. (Provo, UT, USA) from Noni fruit originating in Tahiti. The placebo was prepared using the same procedures and ingredients for making Tahitian Noni ${ }^{\circledR}$ Juice (TNJ), but without the Noni component.

\section{Animals}

Six-week-old female SD rats were purchased from Charles River Inc. (Wilmington, MA, USA). The animals were housed in a room maintained at $25{ }^{\circ} \mathrm{C}$ with a $12 \mathrm{~h}$ photoperiod. They were fed a laboratory chow diet and water ad libitum. The experimental design for these tests was approved by the Institutional Ethics Review Committee for animal experiments at the National Cancer Center.

\section{Experiment 1}

\section{Animal Treatment}

Sixteen female SD rats were divided into two groups of eight each. Both groups were provided drinking water for 7 days. Twenty percent placebo was added to the drinking water in one group and $20 \%$ Noni juice in the other. On day 8 , four animals from each group were fed $0.5 \mathrm{~mL} / \mathrm{kg}$ $\mathrm{CCl}_{4}$ in corn oil. All animals were sacrificed at $6 \mathrm{~h}$ post$\mathrm{CCl}_{4}$ administration. The liver was removed and fixed in $10 \%$ neutral formalin for histological examination. Blood was collected from each animal for serum AST and ALT activity to examine liver function.

\section{LM Examination}

Liver samples were preserved in $10 \%$ neutral buffered formalin and dehydrated in a graded alcohol series. Following xylene treatment, the specimens were embedded in paraffin blocks and cut into $5 \mu \mathrm{m}$ thick sections. Consecutive sections were stained with hematoxylin and eosin (H \& E) and examined under an Olympus BH2 light microscope. The number of foci $/ \mathrm{mm}^{2}$ was counted to assess the degree of necrosis [6]. Digital images were recorded with the Pixera Visual Communication Suite.
Fig. 1 H \& E liver sections (250X), from female SD rats showing protective effect of $20 \%$ Noni pretreatment $\mathbf{d}$ on acute hepatotoxicity at $6 \mathrm{~h}$ post$\mathrm{CCl}_{4}$ exposure $\left(0.5 \mathrm{~mL} / \mathrm{kg} \mathrm{CCl}_{4}\right.$ in corn oil). The rats in $\mathbf{a}$ (control) and $\mathbf{b}$ (noni treated) received no $\mathrm{CCl}_{4}$ exposure, and show no signs of liver-cell damage. With $\mathrm{CCl}_{4}$ exposure, there are significant hepatotoxicity effects: balloon liver cells and apoptotic necrotic cells adjacent to the central vein (arrow and arrowhead (c)). d In contrast, with Noni pretreatment $+\mathrm{CCl}_{4}$ exposure, shows no signs of hepatotoxicity. $\mathbf{b}$ and $\mathbf{d}$ (noni treated) show cell patterns that are more ordered, and show no signs of hepatotoxicity
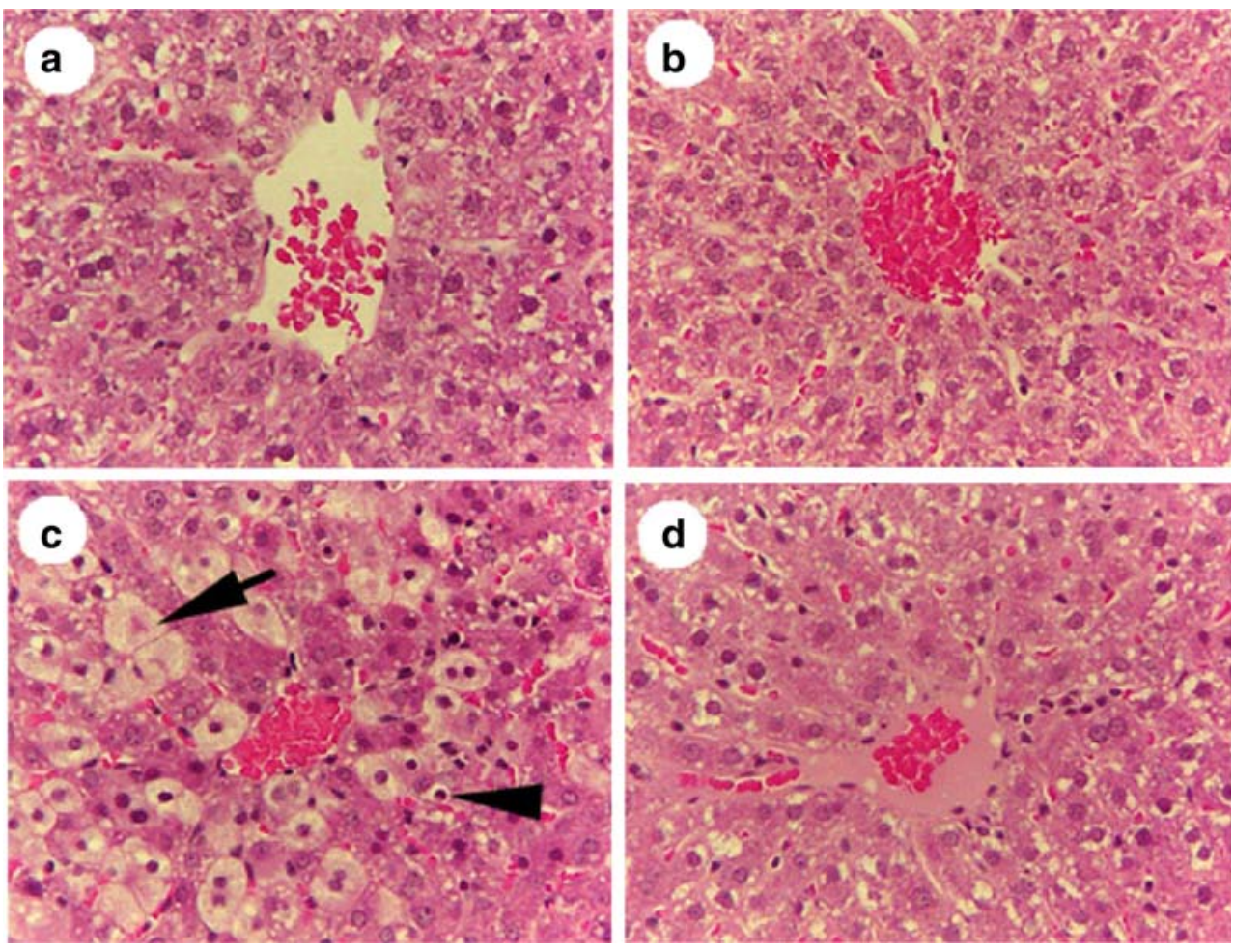


\section{ALT and AST Assay}

Serum was separated from clotted blood by centrifugation at $1,600 \mathrm{rpm}$ for $10 \mathrm{~min}$ at $4{ }^{\circ} \mathrm{C}$. Serum ALT and AST activities were measured using an automatic biochemical analyzer calibrated with quality control standards (E.I. du Pont de Nemours) in a clinical laboratory (Family Medicine Laboratory, UIC College of Medicine at Rockford).

\section{Experiment 2}

\section{Animal Treatments}

Thirty-six female SD rats were divided into two groups of 18 each. While dose range-finding experiments revealed that maximum protection occurred with $20 \%$ Noni juice in drinking water, optimum protection (per amount ingested) occurred with $10 \%$. Therefore, each group was supplied $10 \%$ placebo or $10 \%$ Noni juice in drinking water for 12 days. On day 13, 15 animals from each group were fed $0.25 \mathrm{~mL} / \mathrm{kg}$ $\mathrm{CCl}_{4}$ in corn oil. Three animals from each group were sacrificed at $1,3,6,16$, and $24 \mathrm{~h}$ post- $\mathrm{CCl}_{4}$ administration. The remaining three animals in each group were fed $1 \mathrm{~mL} / \mathrm{kg}$ corn oil and sacrificed $1 \mathrm{~h}$ later, to serve as vehicle placebo and Noni controls. Multiple liver-tissue samples were removed from each rat to be fixed for LM examination.

\section{LM Examination}

Liver samples for experiment 2 were prepared and observed for light microscopy as described in experiment 1 .

\section{Statistical Analysis}

Statistical analysis was done by a Student two-tail $T$ test [7]. Differences were considered as significant at $P<0.05$ and highly significant at $P<0.01$. Results were expressed as mean $\pm \mathrm{SD}$.
Fig. 2 Rat liver sections $(100 \times)$, showing representative sequential hepatopathology at 3 , 6 , or $16 \mathrm{~h}$ following pretreatments with $10 \%$ Noni juice, or placebo, plus $0.25 \mathrm{~mL} / \mathrm{kg} \mathrm{CCl}_{4}$. Panels: a $3 \mathrm{~h}$ post-CCl $\mathrm{Cl}_{4}+\mathrm{Noni}$ juice (normal hepatic structure); b $3 \mathrm{~h}$ post- $\mathrm{CCl}_{4}+$ placebo, (first stages of hepatocyte swelling pericentral to vein; arrow denotes swollen hepatocytes); c $6 \mathrm{~h}$ post- $\mathrm{CCl}_{4}+$ Noni (normal hepatic structure); d $6 \mathrm{~h}$ post$\mathrm{CCl}_{4}+$ placebo, (increased number and degree of swollen hepatocytes); e $16 \mathrm{~h}$ post- $\mathrm{CCl}_{4}+$ Noni (balloon cells adjacent to pericentral vein, and delayed response of hepatotoxicity); and f $16 \mathrm{~h}$ post- $\mathrm{CCl}_{4}+$ placebo (increase in number of damaged foci, and an extension of damaged area midzonal to vein; arrow indicate swollen hepatocytes, arrowhead denotes inflammatory foci)
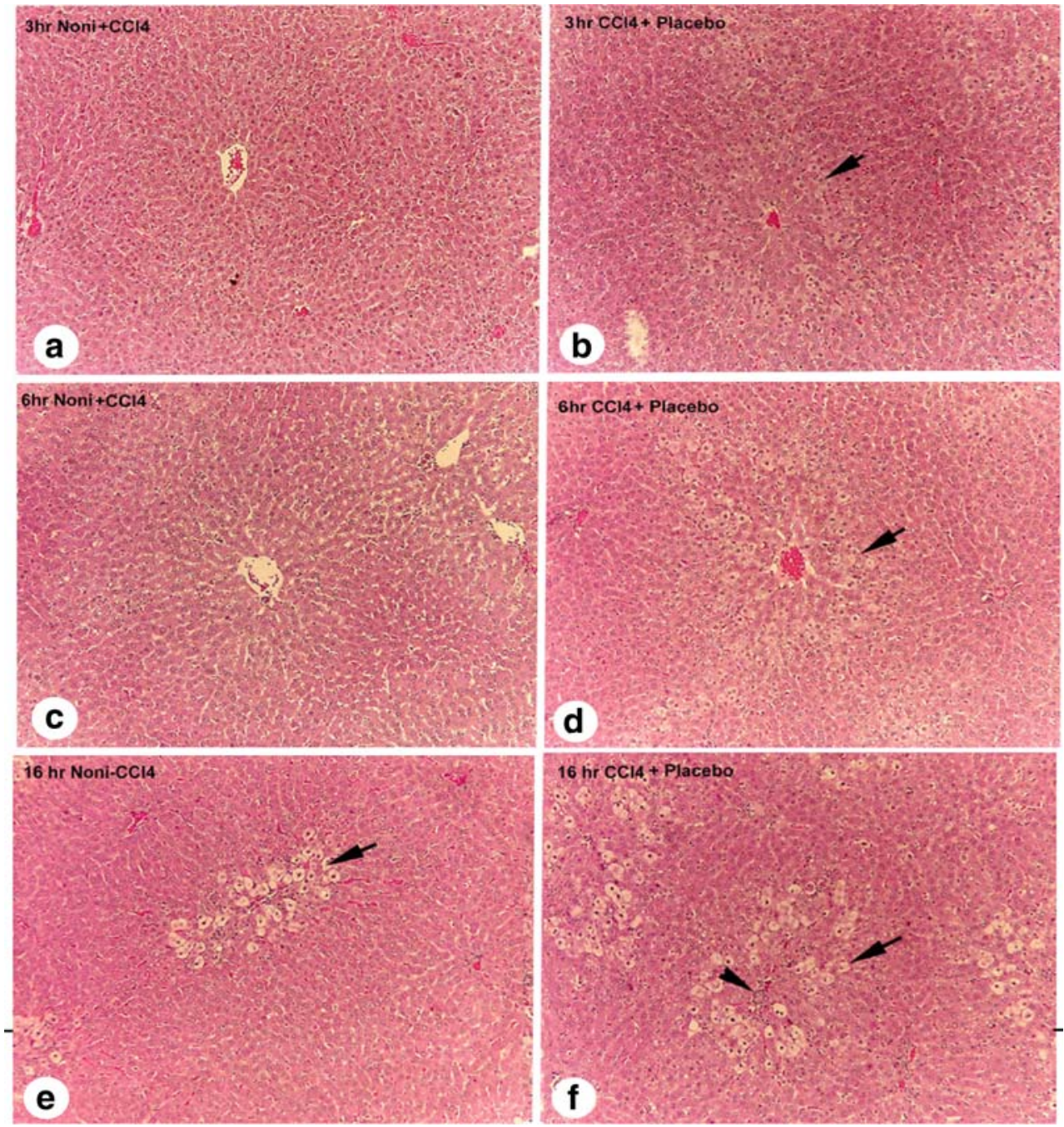
Fig. $3 \mathrm{H} \& \mathrm{E}$ cross sections $(150 \times)$, of pericentral vein areas showing inhibitory effect of $10 \%$ Noni juice on inflammation at $3 \mathrm{~h}$ post- $0.25 \mathrm{~mL} / \mathrm{kg}$ $\mathrm{CCl}_{4}$-induced acute hepatotoxicity (a), versus placebo $+\mathrm{CCl}_{4}$ (b). Arrows denote increased foci of inflammatory cells, arrowheads indicate swollen hepatocytes

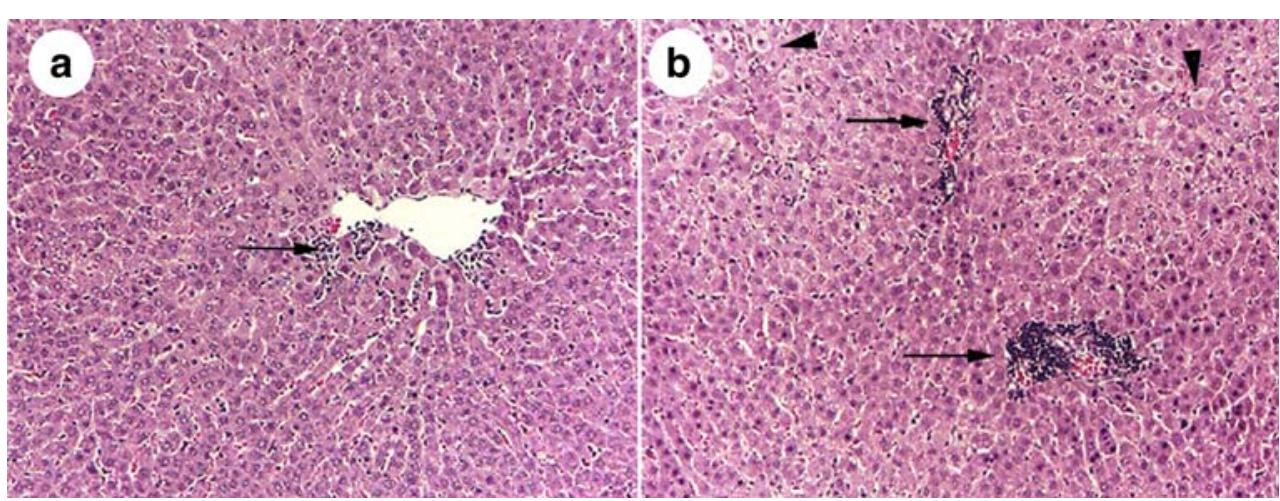

\section{Results and Discussion}

In experiment 1 , the liver lobular structure among rats treated $6 \mathrm{~h}$ earlier with $20 \%$ placebo or $20 \%$ Noni juice did not reveal any noticeable histological changes (Fig. 1a,b), which indicates that high-dose pretreatment with Noni juice or placebo does not produce hepatotoxicity. Hepatic sections, from rats pretreated with $20 \%$ placebo followed by $\mathrm{CCl}_{4}$ treatment, showed typical $\mathrm{CCl}_{4}$-induced liver injury (Fig. 1c). Sections revealed extremely vacuolized hepatocytes (balloon liver cells and apoptotic necrotic cells) adjacent to the central vein (arrows and arrowhead, Fig. 1c), indicating acute $\mathrm{CCl}_{4}$-induced hepatotoxicity. Hepatocytes were significantly protected from $\mathrm{CCl}_{4}$-induced toxicity by $20 \%$ Noni juice pretreatment (Fig. 1d), and demonstrated a normal appearance, except for slight steatosis (fatty deposition) in hepatocytes pericentral to the vein. In the $\mathrm{CCl}_{4}+20 \%$ placebo group, $23.8 \%$ of cross-sectional areas contained swollen balloon cells, whereas in the $\mathrm{CCl}_{4}+20 \%$ Noni group, only $1.9 \%$ contained vacuolized cells $(P<0.001)$. Furthermore, the apoptotic cells were dramatically decreased by $50 \%$ in the Noni pretreated group when compared with the $\mathrm{CCl}_{4}+$ placebo group $(P<0.001)$.

In experiment 2, $10 \%$ Noni $+\mathrm{CCl}_{4}$ pretreatment resulted in a delayed appearance of damaged hepatocytes with a significantly decreased number of necrotic foci $(80 \%)$ and apoptosis $(50 \%)$, compared to the placebo group at 3,6 , and $16 \mathrm{~h}$ (arrows and arrowhead, Fig. 2b,d,f). These results indicate that Noni juice protects the liver from acute $\mathrm{CCl}_{4}$ exposure. Cellular damage consisted of vacuolated hepatocytes and inflammatory cells surrounding the central vein in the placebo $+\mathrm{CCl}_{4}$ group (Fig. 2b,d,f). Conversely, liver lobules in the Noni juice $+\mathrm{CCl}_{4}$ group maintained a normal appearance in each of the post-treatment time intervals (Fig. 2a,c,e).

Comparative histopathological changes of the Noni juice and placebo-treated groups, at $3 \mathrm{~h}$ post $-0.25 \mathrm{~mL} / \mathrm{kg} \mathrm{CCl} \mathrm{Cl}_{4}$ treatment, are shown in Fig. 3. The effects of $10 \%$ Noni juice on reducing inflammation surrounding the central vein area (Fig. 3a), and the absence of swollen hepatocytes are clearly shown in the $\mathrm{H} \& \mathrm{E}$ sections. In Fig. 3b, a similar liver section from the $\mathrm{CCl}_{4}+10 \%$ placebo treatment reveals swollen hepatocytes and a massive influx of inflammatory cells (arrow and arrowheads, Fig. 3a,b).

Noni Juice Suppresses ALT and AST Activities

Serum ALT and AST activities were determined for enzymatic evidence of liver injury (Table 1). The protective effect of Noni juice against $\mathrm{CCl}_{4}$-induced hepatotoxicity was demonstrated by a significant $50 \%$ suppression $(P<$ $0.05)$ of ALT activity compared to the placebo. AST activity in the Noni juice pretreated group was significantly suppressed by $68 \%(P<0.01)$, compared to the placebo. As noted in the table, the $20 \%$ placebo or $20 \%$ Noni juice in drinking water for 7 days did not influence liver function compared with the control. In contrast, the $20 \%$ placebo + $\mathrm{CCl}_{4}$ treatment produced acute liver damage as measured by elevated serum ALT and AST activities.

The present study supports the protective effects of Noni juice on acute $\mathrm{CCl}_{4}$-induced hepatotoxicity. Necrosis of hepatocytes was minimized in livers of animals pretreated with Noni juice in both experiments. Noni juice prevented free-radical-induced oxidative-pathological events.

Damaged hepatocytes release ALT and AST enzymes following acute exogenous toxin exposure. Therefore these elevated serum enzyme levels are good chemical indicators of liver damage. Noni juice is able to normalize liver function after

Table 1 Effect of Noni juice on acute $\mathrm{CCl}_{4}$-induced hepatitis in $\mathrm{SD}$ rats

\begin{tabular}{lcc}
\hline Treatment & ALT (IU/L) & AST (IU/L) \\
\hline Control & $67 \pm 31^{* *}$ & $222 \pm 121^{* *}$ \\
Placebo (20\%) & $67 \pm 26^{* *}$ & $222 \pm 99^{* *}$ \\
Noni juice (20\%) & $65 \pm 59^{* *}$ & $184 \pm 31^{* *}$ \\
Placebo $(20 \%)+\mathrm{CCl}_{4} 0.5 \mathrm{~mL} / \mathrm{kg}^{\mathrm{a}}$ & $136 \pm 41$ & $1135 \pm 466$ \\
Noni juice $(20 \%)+\mathrm{CCl}_{4} 0.5 \mathrm{~mL} / \mathrm{kg}$ & $68 \pm 12^{* *}$ & $358 \pm 69^{*}$ \\
\hline
\end{tabular}

${ }^{a}$ Data from each group were compared to that of the positive control group $\left(20 \%\right.$ placebo $\left.+0.5 \mathrm{~mL} / \mathrm{kg} \mathrm{CC1}_{4}\right)$. Data are expressed as means $\pm \mathrm{SD}(n=4)$. Student two-tail $T$ test

${ }^{*} P<0.05,{ }^{* *} P<0.01$ 
acute exposure to $\mathrm{CCl}_{4}$. Serum ALT and AST activity, a measure of defense enzyme levels, was significantly suppressed after $\mathrm{CCl}_{4}$ exposure in the Noni juice pretreated animals, compared with the elevated enzyme levels in the placebo pretreated animals. Therefore, pretreatment with Noni juice indeed protected hepatocytes from acute $\mathrm{CCl}_{4}$ exposure.

A few reports have suggested the involvement of Noni juice in the development of chemically induced hepatitis in a limited number of cases in Europe. However, no causal role has been established in any of these instances. Further, an official European investigation of these cases determined that no relationship between Noni juice and hepatitis was evident and that consumption of Noni juice is unlikely to induce adverse human liver effects [8]. Animal and human studies have revealed that Noni juice is not hepatotoxic, even at very high doses $[9,10]$.

\section{Conclusions}

Our investigation suggests that Noni juice exerts effective protection against acute extrinsic chemical induced hepatic injury by inhibiting inflammatory response and suppressing elevated liver enzyme activities; thus preventing consequent cell membrane damage. Histopathological observations revealed morphological evidence, and bioassays revealed functional evidence, that Noni confers resistance to acute $\mathrm{CCl}_{4}$-induced hepatotoxicity. High dosages of Noni and placebo pretreatments did not induce liver damage. Noni may thus be an efficacious natural hepatoprotective nutritional supplement against chemically induced hepatotoxicity. Future studies should focus on how Noni juice influences the inflammatory and cytotoxic cytokines involved in liver injury.

Acknowledgements This study was financially supported by Morinda Holding Inc. We thank Drs. Richard Novak, University of Illinois, and Leland White, Morinda Holdings, Inc., Provo, UT, USA for the editing and preparation of the manuscript. We thank technicians in the clinical laboratory of Family Health Center of UIC College of Medicine for their technical help in the liver function tests. A special thanks to Mr. John Javaherian for his effort on animal care.

Open Access This article is distributed under the terms of the Creative Commons Attribution Noncommercial License which permits any noncommercial use, distribution, and reproduction in any medium, provided the original author(s) and source are credited.

\section{References}

1. Recknagel RO (1967) Carbon tetrachloride hepatotoxicity. Pharmacol Rev 19:145-208

2. Liu SL, Degli Esposti S, Yao T, Diehl AM, Zern MA (1995) Vitamin $\mathrm{E}$ therapy of acute $\mathrm{CCl}_{4}$-induced hepatic injury in mice is associated with inhibition of nuclear factor kappa B binding. Hepatology 22:1474-1481

3. Seifert WF, Bosma A, Hendriks HF, van Leeuwen RE, van Thiel-de Ruiter GC, Seifert BI, Knook DL, Brouwer A (1995) Beta-carotene (provitamin A) decreases the severity of $\mathrm{CCl}_{4}$-induced hepatic inflammation and fibrosis in rats. Liver 15:1-8

4. Aniya Y, Koyama T, Miyagi C, Miyahira M, Inomata C, Kinoshita S, Ichiba $\mathrm{T}$ (2005) Free radical scavenging and hepatoprotective actions of the medicinal herb, Crassocephalum crepidioides from the Okinawa Islands. Biol Pharm Bull 28:19-23

5. Wang MY, Su C (2001) Cancer preventive effect of Morinda citrifolia (Noni). Ann NY Acad Sci 952:161-168

6. Brattin WJ, Glende EA, Recknagel RO (1985) Pathological mechanisms in carbon tetrachloride hepatotoxicity. Free Radic Biol Med 1:27-38

7. Neely JG, Hartman JM, Forsen JW Jr, Wallace MS (2003) Tutorials in clinical research: VII. Understanding comparative statistics (contrast) - part B: application of $T$-test, Mann-Whitney $U$, and chi-square. Laryngoscope 113:1719-1725

8. EFSA European Food Safety Authority (2006) Opinion on a request from the Commission related to the safety of Noni juice (juice of the fruits of Morinda citrifolia). EFSA J 376:1-12

9. West BJ, Jensen CJ, Westendorf J, White LD (2006a) A safety review of noni fruit juice. J Food Sci 71:R100-R106

10. West BJ, Jensen CJ, Westendorf J (2006b) Noni juice is not hepatotoxic. World J Gastroenterol 12:3616-3619 